\title{
CHANGE OF FREE VOLUME IN POLYMER GELS AS STUDIED BY POSITRON ANNIHILATION LIFETIMES
}

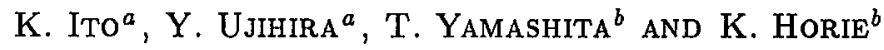 \\ ${ }^{a}$ Research Center for Advanced Science and Technology, The University of Tokyo \\ 4-6-1 Komaba, Meguro-ku, Tokyo 153-8904, Japan \\ ${ }^{6}$ Graduate School of Engineering, The University of Tokyo \\ 7-3-1 Hongo, Bunkyo-ku, Tokyo 113-8656, Japan
}

\begin{abstract}
Variations of a free volume size of a poly $(N$-isopropylacrylamide) gel and a polyacrylamide gel during a volume phase transition induced by a change of external environment - temperature, solvent composition, $\mathrm{pH}$, and ion strength, are described. The free volume size is probed by positron annihilation lifetime technique. Variations of the free volume size suggest that a nanoscopic environment of the gels is affected by the free volume of the solvent phase and an interaction between the polymer chain and the solvent molecule in the swollen state, and by the aggregation of the polymer chain and a balance of the interaction among the polymer chain and the solvent molecules in the collapsed state. It is revealed that the free volume size reflects the essential change of the nanoscopic environment in the gels which could induce the macroscopic volume phase transition.
\end{abstract}

PACS numbers: $82.70 . \mathrm{Gg}$

\section{Introduction}

Volume phase transition (VPT) of polymer gels, such as a poly $(N$-isopropylacrylamide) (PNIPA) gel, a polyacrylamide (PAAm) gel, has been studied to clarify the phenomenon for its scientific, technological and practical importance [1-3]. The VPT was interpreted from the macroscopic point of view both empirically and theoretically employing statistical thermodynamical theory for a polymer solution developed by Flory and Huggins. In the present paper, the VPT is studied from the nanoscopic point of view utilizing the free volume hole sizes of the PNIPA and PNIPA gels obtained by positron annihilation lifetime technique. The change of a subnano-environment in the gels, and an origin and a location of the free volumes are discussed in the continuous or discontinuous VPTs induced by the various physico-chemical stimuli such as temperature (Fig. 1a), solvent composition (Figs. 1b and c), pH (Fig. 1d) and ion strength (Fig. 1e). A relation between the free volume size and the macroscopic volume change is predicted in terms of the interaction between the polymer chain and the solvent molecules. 

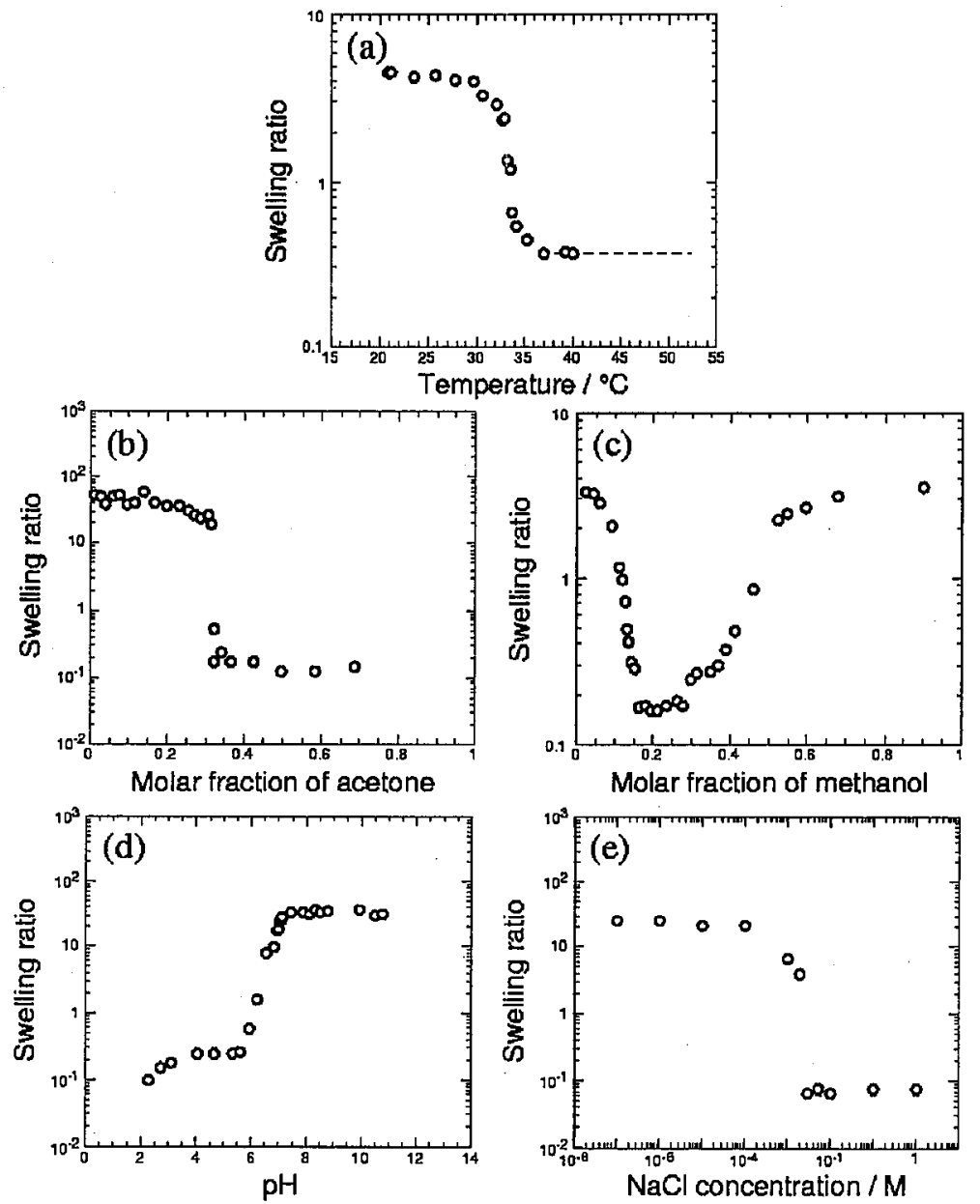

Fig. 1. Swelling curves of polyacrylamide (PAAm) and poly $(N$-isopropylacrylamide) (PNIPA) gels as a function of external physico-chemical stimuli; (a) temperature, (b) and (c) solvent composition, (d) $\mathrm{pH}$ and (e) ionic strength. The measurements were performed at $20^{\circ} \mathrm{C}$ with exception of temperature dependence. (a) The data for the PNIPA gel in water, (b) for the PAAm gel [carboxylate content: $4 \mathrm{~mol} \%$ ] in the acetone-water mixed solvent, (c) for the PNIPA gel in the methanol-water mixed solvent, (d) for the PAAm gel [2 mol\%] in the acetone-water $1: 1(\mathrm{v} / \mathrm{v})$ mixed solvent, (e) for the PAAm gel [4 mol\%] in the acetone-water $3: 2(\mathrm{v} / \mathrm{v})$ mixed solvent.

\section{Experimental}

The PAAm and PNIPA gels were prepared by the radical copolymerization of acrylamide for the PAAm gel or $N$-isopropylacrylamide for the PNIPA gel and $N, N^{\prime}$-methylenebisacrylamide cross-linker. Sodium acrylate was added to a solution for the PAAm gel as an ionic agent. The amount of the ionic agent was regulated to make carboxylate residue content 2 or $4 \mathrm{~mol} \%$. The copolymerizations were. 
performed by a conventional manner using tetramethylenediame accelerator and ammonium persulfate initiator at room temperature. Prepared gels were washed after the copolymerization, then, the degree of swelling equilibrium was measured (Fig. 1). Details of the preparation are found in previous literatures $[4,5]$.

Decay curves of the positron annihilation were recorded with a conventional fast-fast coincident system of a time resolution of $290 \sim 300 \mathrm{ps}$. The positron source was $7.5 \times 10^{5} \mathrm{~Bq}{ }^{22} \mathrm{Na}$ sealed in Kapton foils. The lifetime measurement for the temperature dependence was performed in the temperature range from 20 to $50^{\circ} \mathrm{C}$, while the other measurements were done at $20^{\circ} \mathrm{C}$. The obtained decay curves of positrons with total counts of $1 \sim 8$ million were fitted by a non-linear least-square method and resolved into three or four components by using the PATFIT-88 computer program with a good statistical precision. The free volume hole size, $R[\mathrm{~nm}]$, for the PAAm and PNIPA gels was estimated by using the following Nakanishi-Jean equation [6]:

$$
\tau=0.5\left[1-\frac{R}{R+0.166}+\frac{1}{2 \pi} \sin \left(\frac{2 \pi R}{R+0.166}\right)\right]^{-1},
$$

where $\tau[\mathrm{ns}]$ represents an annihilation lifetime of ortho-positronium (o-Ps). It should be considered that the "Ps bubble" or Ps cavity could be expected in a liquid and a solution such as the hydrogel. The relation between the "Ps bubble" size and a free volume size obtained by the velocity of sound was studied by Ujihira et al. for various organic liquids [7]. Good correlation between them was revealed. Therefore, we conclude that a change of the Ps cavity size reflects that of the free volume size for the PAAm and PNIPA gel.

\section{Results and discussion}

\subsection{Free volume hole size in macroscopically swollen and collapsed state}

\subsubsection{Swollen state}

Free volume sizes of the swollen gels are close to those of the corresponding mixed solvent. The result may be due to a reduced concentration of the polymer chains in the gels. In other words, most of the Ps atoms annihilate in the free volumes present in the solvent phase, suggesting that the nanoscopic structure of the solvent in the gel-network is similar to that of the bulk solvent. However, a significant discrepancy is observed in the PNIPA gel swollen in the methanol rich solvent. Two types of free volumes are appeared in the region. A radius of the smaller free volume is $\sim 0.25 \mathrm{~nm}$.

\subsubsection{Collapsed state}

The free volume sizes decreased with the volume shrinkage of the PAAm gels (Figs. $2 \mathrm{~b}, \mathrm{~d}$ and $\mathrm{e}$ ). This suggests that the aggregation of the polymer chain significantly influences the free volume size in the PAAm gels. It could be speculated that a restriction of a local movement of the polymer chain of the PAAm. occurred. On the other hand, the free volume of the collapsed PNIPA gel induced by temperature change is divided into two components, bigger and smaller free volume than that in the swollen PNIPA gel (Fig. 2a), while the free volume size in the collapsed PNIPA gel induced by the compositional change of methanol-water 

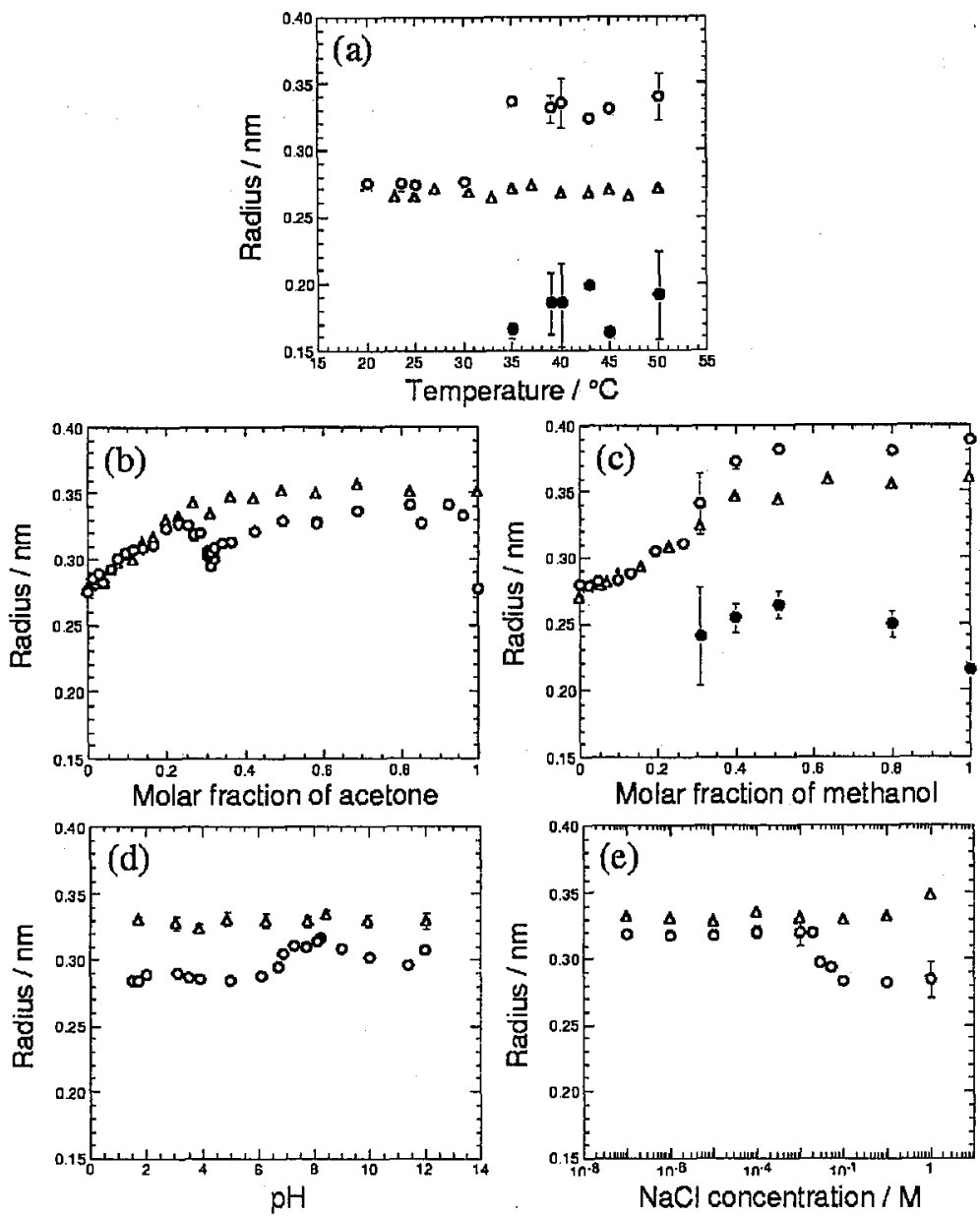

Fig. 2. Variations of the free volume radius for the PAAm and PNIPA gels $(\bullet ; 3 a$ component, $O ; 3 \mathrm{~b}$ component) and the corresponding solvent $(\triangle)$ as a function of the external physico-chemical stimuli. A detailed illustration of the data is shown in the caption for Fig. 1.

agrees with that of the corresponding mixed solvent (Fig. 2c). The peculiar result of the collapsed PNIPA gel by the temperature change may be caused by the presence of periodically arrayed small free volume detected by a delocalized Ps atom (Bloch state of Ps).

\subsection{Origin of the macroscopic volume change of the hydrogels probed by free volume}

Average free volume sizes of the PAAm and PNIPA gels are listed in Table as a function of a stimulus variable. As mentioned above, the free volume size of the swollen gels generally agrees with that of the corresponding solvent, while that 
of the collapsed gels decreases with the macroscopic volume shrinkage except the case of the solvent composition dependence. Two kinds of the free volumes were observed for the PNIPA gels.

TABLE

Variations of a free volume of polyacrylamide and poly $(N$-isopropylacrylamide) gels during a volume phase transition.

\begin{tabular}{|c|c|c|c|c|}
\hline \multirow[t]{2}{*}{$\begin{array}{l}\text { Stimulus } \\
\text { variable }\end{array}$} & \multirow{2}{*}{$\begin{array}{c}\text { Chemical } \\
\text { constitution of } \\
\text { the gel-network } \\
\text { (Carboxylate } \\
\text { content/mol\%) }\end{array}$} & \multirow[t]{2}{*}{ Solvent } & \multicolumn{2}{|c|}{$\begin{array}{l}\text { Average free } \\
\text { volume } \\
\text { radius/nm }\end{array}$} \\
\hline & & & $\begin{array}{c}\text { Swollen } \\
\text { state }\end{array}$ & $\begin{array}{c}\text { Collapsed } \\
\text { state }\end{array}$ \\
\hline Temperature & $\begin{array}{c}\text { PNIPA } \\
\text { (non-ionized) }\end{array}$ & Water & 0.274 & $0.182,0.333$ \\
\hline \multirow[b]{2}{*}{$\begin{array}{c}\text { Solvent } \\
\text { composition }\end{array}$} & PAAm (4) & Acetone-water & 0.304 & 0.319 \\
\hline & $\begin{array}{c}\text { PNIPA } \\
\text { (non-ionized) }\end{array}$ & $\begin{array}{c}\text { Methanol-- } \\
\text { water }\end{array}$ & $\begin{array}{c}0.215^{*} \\
0.388^{*} \\
0.274^{* *}\end{array}$ & 0.316 \\
\hline $\mathrm{pH}$ & PAAm (2) & $\begin{array}{c}\text { Acetone- } \\
\text { water }[1: 1(\mathrm{v} / \mathrm{v})]\end{array}$ & 0.307 & 0.286 \\
\hline $\begin{array}{c}\text { Ionic } \\
\text { strength }\end{array}$ & PAAm (4) & $\begin{array}{c}\text { Acetone- } \\
\text { water }[3: 2(\mathrm{v} / \mathrm{v})]\end{array}$ & 0.319 & 0.288 \\
\hline
\end{tabular}

${ }^{*}$ Methanol rich phase. ${ }^{* *}$ Water rich phase.

Free volume radius ranging from $0.2 \mathrm{~nm}$ to $0.3 \mathrm{~nm}$ in polymers and solutions, which have an interaction through the hydrogen bond, is often observed in the positron annihilation lifetime. The free volume size of $\sim 0.29 \mathrm{~nm}$ in radius was observed in the collapsed PAAm gels by the $\mathrm{pH}$ and ionic strength change. This implies that the hydrogen bonding plays an important role of the aggregation of the gel-network. On the other hand, the free volume size of $\sim 0.32 \mathrm{~nm}$ in radius is observed in the collapsed gel by the solvent composition change, which is larger than that obtained by the changes of the $\mathrm{pH}$ and ionic strength. The evidence that the free volume size is close to the corresponding mixed solvent, suggests that a balance of the interaction among the solvent molecules and the polymer chain also affects the aggregation of the gel-network, i.e., the macroscopic volume shrinkage.

Hydrophobicity of the PNIPA chain is higher than that of the PAAm chain due to a presence of the isopropyl group. The volume change of the PNIPA gel at $33^{\circ} \mathrm{C}$ in water is interpreted to be due to a destruction of the hydrogen bonding between the polymer chain and the water molecule and the aggregation of the polymer chain by the hydrophobic interaction. As a result of the shrinkage, the periodically arrayed smaller free volumes due to bound water molecules in the aggregated gel-network were observed. The free volume size of the methanol-water mixed solvent increased with an increase in a methanol fraction because of the hydrophobicity of the methanol molecule. The hydrogen bonding between the 
polymer chain and the solvent molecule is produced in the swollen PNIPA gel by the methanol rich solvent so that the smaller free volumes were observed. It is expected that a formation of the free volume due to the hydrophobic interaction resulted in the macroscopic volume change of the PNIPA gel with the consequence that a supplemental phase by the hydrogen bond appeared.

The free volume of the swollen PAAm and PNIPA gels is influenced not only by the interaction between the polymer chain and the solvent molecule, but that in the solvent phase. The free volume of the collapsed gels is influenced by the aggregation of the polymer chain and the interactions among the polymer chain and the solvent molecules.

\section{Conclusion}

The positron annihilation lifetime technique is advantageously utilized to monitor the nanoscopic environment of the PAAm and PNIPA gels during the VPT. The correlation between the free volume size and the state of the interactions among the polymer chain and the solvent molecules in the gels was revealed. The present results prove that the free volume size reflects the nanoscopic environmental change in the gels, which induces the macroscopic VPT.

\section{Acknowledgment}

This work is supported by Research Fellowships of the Japan Society for the Promotion of Science for young scientists.

\section{References}

[1] Gel Handbook, Eds. Y. Nagata, K. Kajiwara, N.T.S. Co. Ltd., Tokyo 1997.

[2] Advances in Polymer Science, Responsive Gels: Volume Transitions I, Ed. K. Dušek, Vol. 109, 110, Springer-Verlag, Berlin 1993.

[3] T. Tanaka, Sci. Am. 244, 110 (1981).

[4] K. Ito, Y. Ujihira, T. Yamashita, K. Horie, J. Polym. Sci., Part B: Polym. Phys. 36, 1141 (1998).

[5] K. Ito, Y. Ujihira, T. Yamashita, K. Horie, Mater. Sci. Forum 255-257, 375 (1997).

[6] H. Nakanishi, S.J. Wang, Y.C. Jean, in: Int. Symp. on Positron Annihilation Studies of Fluids, Ed. S.C. Sharma, World Scientific, Singapore 1987, p. 292.

[7] Y. Ujihira, T. Ryuo, Y. Kobayashi, T. Nomizu, Appl. Phys. 16, 71 (1987). 